\title{
Conectividad entre los Andes y la Amazonía, un análisis de la configuración del paisaje del departamento de Caquetá, Colombia
}

Recepción: 3 de junio de 2021 - Evaluación: 15 de diciembre de 2021 • Aprobación: 17 de diciembre de 2021

Resumen: En este trabajo se analizó cómo la configuración de los elementos del paisaje del departamento del Caquetá, al sur de Colombia, permite o restringe la propagación de flujos involucrados en la conectividad ecológica entre los Andes y la Amazonía, ya que este departamento se encuentra en la zona transicional de estas dos regiones. Desde el enfoque de la ecología del paisaje, se agruparon los elementos que componen este paisaje en cinco sistemas planetarios: atmosférico, hidrosférico, geosférico, biosférico y antroposférico. Se valoraron las condiciones de los elementos seleccionados por cada sistema asignándole un costo de $1 \mathrm{a}$ 10 para posteriormente representarlos por medio de una superficie de costo, lo cual permite comprender de manera sistémica la dinámica de conectividad y facilita saber dónde y de qué manera la configuración del paisaje restringe o favorece el movimiento de los flujos, las relaciones o los procesos ecológicos. Esta superficie de costo permitió entender que la configuración del paisaje del departamento en un 83\% de su área permite la conectividad con un alto movimiento de flujos entre los Andes y la Amazonia colombiana.

Palabras clave: ecología del paisaje, conectividad, sistemas planetarios, flujos, paisaje, superficie de costo.

Para citar: Rusinque Quintero, L. L., Moyano Molano, A. L., \& Montoya Rojas, G. A. (2022). Conectividad entre los Andes y la Amazonía, un análisis de la configuración del paisaje del departamento de Caquetá, Colombia. Perspectiva Geográfica, 27(1), 86-105. https://doi. org/10.19053/01233769.12944

Especialista en gestión y planificación del desarrollo urbano y regional, Escuela Superior de Administración Pública (ESAP), Ingeos SAS, Bogotá, Colombia. Correo: lindarusinque@saltamontesoutdoor.com. CVLAC: https://scienti.minciencias.gov. co/cvlac/visualizador/generarCurriculoCv.do?cod_rh=0001790829\&lang=es. Orcid: https://orcid.org/0000-0003-1999$786 \mathrm{X}$

Ingeniera geógrafa y ambiental, Universidad de Ciencias Aplicadas y Ambientales (UDCA), Ingeos sAs, Bogotá, Colombia. Correo: angiemoyano@saltamontesoutdoor.com cvLAC: https://scienti.minciencias.gov.co/cvlac/visualizador/generarCurriculoCv.do?cod_rh=0001805712\&lang=es. Orcid: https://orcid.org/0000-0002-1084-5192 


\title{
Connectivity between the Andes and the Amazon, an analysis of the landscape configuration of the department of Caquetá, Colombia
}

\begin{abstract}
This paper analyzed how the configuration of landscape elements in the department of Caquetá, southern Colombia, allows or restricts the spread of flows involved in the ecological connectivity between the Andes and the Amazon, since this department is located in the transitional zone of these two regions. From a landscape ecology approach, the elements that make up this landscape were grouped into five planetary systems: atmospheric, hydrospheric, geospheric, biospheric and anthropospheric. The conditions of the elements selected for each system were assessed by assigning them a cost from 1 to 10 and then representing them by means of a cost surface, which allows a systemic understanding of the dynamics of connectivity and makes it easier to know where and in what way the configuration of the landscape restricts or favors the movement of flows, relationships or ecological processes. This cost surface allowed us to understand that the configuration of the landscape of the department in $83 \%$ of its area allows connectivity with a high movement of flows between the Andes and the Colombian Amazon.
\end{abstract}

Keywords: landscape ecology, connectivity, planetary systems, flows, landscape, cost surface.

\section{Conectividade entre os Andes e a Amazônia, uma análise da configuração da paisagem do departamento de Caquetá, Colômbia}

\begin{abstract}
Resumo: Neste trabalho se analisou como a configuração dos elementos da paisagem do departamento de Caquetá, no sul da Colômbia, permite ou restringe a propagação de fluxos envolvidos na conectividade ecológica entre os Andes e a Amazônia, já que este departamento se encontra na zona transicional destas duas regiões. Desde a abordagem da ecologia da paisagem, agruparam-se os elementos que compõem esta paisagem em cinco sistemas planetários: atmosférico, hidrosférico, geosférico, biosférico e antroposférico. Foram valorizadas as condições dos elementos selecionados por cada sistema atribuindo-lhe um custo de 1 a 10 para posteriormente representá-los por meio de uma superfície de custo, o que permite uma compreensão de maneira sistémica da dinâmica de conectividade e facilita saber onde e de que maneira a configuração da paisagem restringe ou favorece a circulação de fluxos, as relações ou os processos ecológicos. Esta superfície de custo permitiu entender que a configuração da paisagem do departamento em 83\% de sua área permite a conectividade com um alto movimento de fluxos entre os Andes e a Amazônia colombiana.
\end{abstract}

Palavras-chave: ecologia da paisagem, conectividade, sistemas planetários, fluxos, paisagem, superficie de custo. 


\section{Introducción}

El paisaje es una expresión integrada por la relación ser humano-naturaleza representada en un sistema que adquiriere sus características tras un largo periodo de tiempo (Vásquez \& Andrade, 2016), dando lugar a áreas geográficas heterogéneas con diversos elementos que interactúan entre sí (Vila et al., 2006; Wu, 2019). Entonces, la ecología del paisaje busca también integrar al máximo dichos elementos, sean bióticos o abióticos, desde un análisis sistémico que permite comprender de una forma más aproximada a la realidad sus diferentes procesos (Vila et al., 2006). Von Bertalanffy (1993) coincide con la idea de abarcar este estudio como un sistema en el que se comprenden sus componentes y esto, a su vez, permite ver diferencias con otros sistemas, pero que, de manera conjunta, dos o más sistemas operan como un todo complejo determinando así los diferentes procesos en un paisaje.

El paisaje es un sistema compuesto por subsistemas divididos en componentes que contienen elementos que determinan la conectividad del paisaje, puesto que es su configuración la que permite o restringe la dispersión de los flujos ecológicos (Gurrutxaga \& Lozano, 2008; Taylor et al., 1993).

Se abordaron cinco sistemas según la clasificación y definición de Montoya-Rojas (2018) y Montoya-Rojas et al. (2019), lo cual permitió analizar el comportamiento complejo de las relaciones entre los elementos y los componentes. El primer sistema, el atmosférico, engloba las variables meteorológicas y su dinámica tanto en el espacio como en el tiempo, seguido por el hidrosférico, donde se relaciona la dinámica e interacción entre los cuerpos de agua tanto superficiales como subterráneos; en el geosférico se observa las dinámicas geológicas, geomorfológicas y edafológicas; el biosférico está basado en la interrelación de la fauna y la flora y, por último, el sistema antroposférico, que corresponde al medio geográfico y social en el que se desarrolla la vida humana y cada una de sus actividades.
El impacto negativo de la pérdida de conectividad en el paisaje se acumula lentamente, demostrando que las pérdidas van más allá del momento concreto en el que se cambió la configuración original del paisaje (Saura, 2013). Es por esto que es importante analizar los flujos en el contexto del paisaje por donde se mueven y con esto aportar en los esfuerzos para la conservación de la naturaleza y la biodiversidad, contribuyendo así a la mitigación de los efectos del cambio climático (Crooks \& Sanjayan, 2006, citado en Saura, 2013).

Para lo anterior se realizaron cinco matrices y una superficie de costo, las cuales son un producto del análisis cartográfico de diferentes insumos geográficos utilizando un sistema de información geográfica (SIG). La superficie representa la permeabilidad del paisaje a los flujos analizados, donde cada píxel tiene un valor referente a dicha permeabilidad (Correa-Ayram, 2017; Saura et al., 2011); por lo tanto, la superficie de costo permite identificar los lugares donde los diferentes flujos del paisaje son más o menos favorecidos (Balbi et al., 2019).

El departamento de Caquetá hace parte de la cuenca hidrográfica del río Amazonas, se encuentra en la zona transicional del sistema Andes-Amazonía y es un escenario ecológico con características especiales por ser la zona donde interactúan los ecosistemas montañosos de los Andes y la selva húmeda tropical amazónica en Colombia (Clerici et al., 2019; Comisión Económica para América Latina y el Caribe [CEPAL] \& Patrimonio Natural, 2013). Sin embargo, este departamento ha sido protagonista de distintas olas extractivistas y dinámicas productivas que han modelado el paisaje actual (Ciro-Rodríguez, 2018), entre otras, la extracción de quina a finales del siglo XIX, del caucho a principios del siglo Xx, luego, en los años treinta, la ganadería y a finales de los años setenta los cultivos de coca. En los últimos 40 años se ha incrementado la presión antrópica por la expansión agropecuaria, traducida en el agotamiento y la contaminación de recursos naturales, la pérdida acelerada de biodiversidad, la erosión, la degradación del suelo y la deforestación (Clerici et al., 2019). 
En cuanto a la deforestación en el departamento, el Instituto de Hidrología, Meteorología y Estudios Ambientales (IDEAM), en sus boletines trimestrales de alertas tempranas de deforestación, menciona los principales núcleos de pérdida de bosque en Colombia: para el 2017, registró que 12 de los 24 núcleos se encontraban en la región amazónica, donde Caquetá es el departamento con el puesto de mayor porcentaje de hectáreas deforestadas (28\%, con 60.373 ha), especialmente en los municipios de San Vicente de Caguán y Cartagena de Chairá, ubicados en el piedemonte de la región (IDEAM, 2017). Para el 2018, el IDEAM reportó una disminución del 10\% de la deforestación nacional respecto al año anterior; sin embargo, el Amazonas presentó el valor más alto, con un 70,1\% de la totalidad de deforestación del país, y Caquetá nuevamente ocupó la misma posición, con 46.765 ha deforestadas, sumando a la lista el municipio de Solano. Finalmente, para el último trimestre del 2019, el IDEAM (2019a) reportó que en Caquetá se encontraba el 26\% del área deforestada del país concentrada en los tres municipios nombrados, en especial dentro de los parques nacionales naturales Serranía de Chiribiquete y Cordillera de los Picachos.

\section{Metodología}

\section{1. Área de estudio}

Aun cuando la ecología del paisaje no responde a delimitaciones político-administrativas (Serrano, 2012), se toma como área de estudio el departamento de Caquetá por su ubicación (Figura 1) entre los Andes y la Amazonía al extenderse desde la cordillera Oriental hasta la selva amazónica, incluyendo la Serranía de Chiribiquete (CEPAL \& Patrimonio Natural, 2013). Caquetá está localizado al sur de Colombia, cuenta con un área de $90.108 \mathrm{~km}^{2}$ y es el tercer departamento más grande del país, ya que cubre aproximadamente el 8\% de su área continental. Cuenta con una población de 359.602 habitantes (Departamento Administrativo Nacional de Estadística [DANE], 2018) y administrativamente está divido en 16 municipios, de los cuales los de mayor extensión son San Vicente del Caguán, Cartagena del Chairá y Solano. Sobre estos tres municipios se encuentra el 75\% del área total del Parque Nacional Natural (PNN) Serranía de Chiribiquete, el área protegida más extensa del país.

\subsection{Matrices de costo}

Saura et al. (2011) y Saura (2013) afirman que la conectividad debe medirse como un aspecto funcional que incluye la influencia de la configuración del paisaje en la conectividad de los fragmentos que se desean evaluar, primero, analizando distintos flujos ecológicos por separado para posteriormente integrar todos los resultados para identificar las zonas del paisaje más relevantes en la conectividad y reconocer la localización de los obstáculos que impiden su propagación. Para esto se elaboró una superficie de costo que permite identificar de manera aproximada las áreas en donde es o no favorecida la conectividad del paisaje (Balbi et al., 2019; Saura, 2013). Esta superficie se generó a partir de la ponderación de cinco matrices de costo resultantes, una por cada sistema planetario.

Las matrices de costo son el resultado del análisis de conectividad de los diferentes elementos geográficos agrupados por cada sistema planetario y se construyeron a partir de los valores asignados a cada píxel correspondiente al costo energético que le supone a un flujo recorrer este espacio. En este caso, dichos valores fueron adaptados de la metodología de Beier et al. (2009), donde se utiliza una escala de 1 a 10 con cuatro intervalos (Tabla 1). Esta valoración se realizó por cada sistema planetario según los elementos considerados como influyentes en la propagación de flujos, como desarrollan Moyano-Molano y Rusinque-Quintero (2020). La escala para construir estas matrices en función de los criterios de análisis e insumos disponibles fue 1:100.000 y generaron un estudio semidetallado (Montoya-Rojas, 2018). 


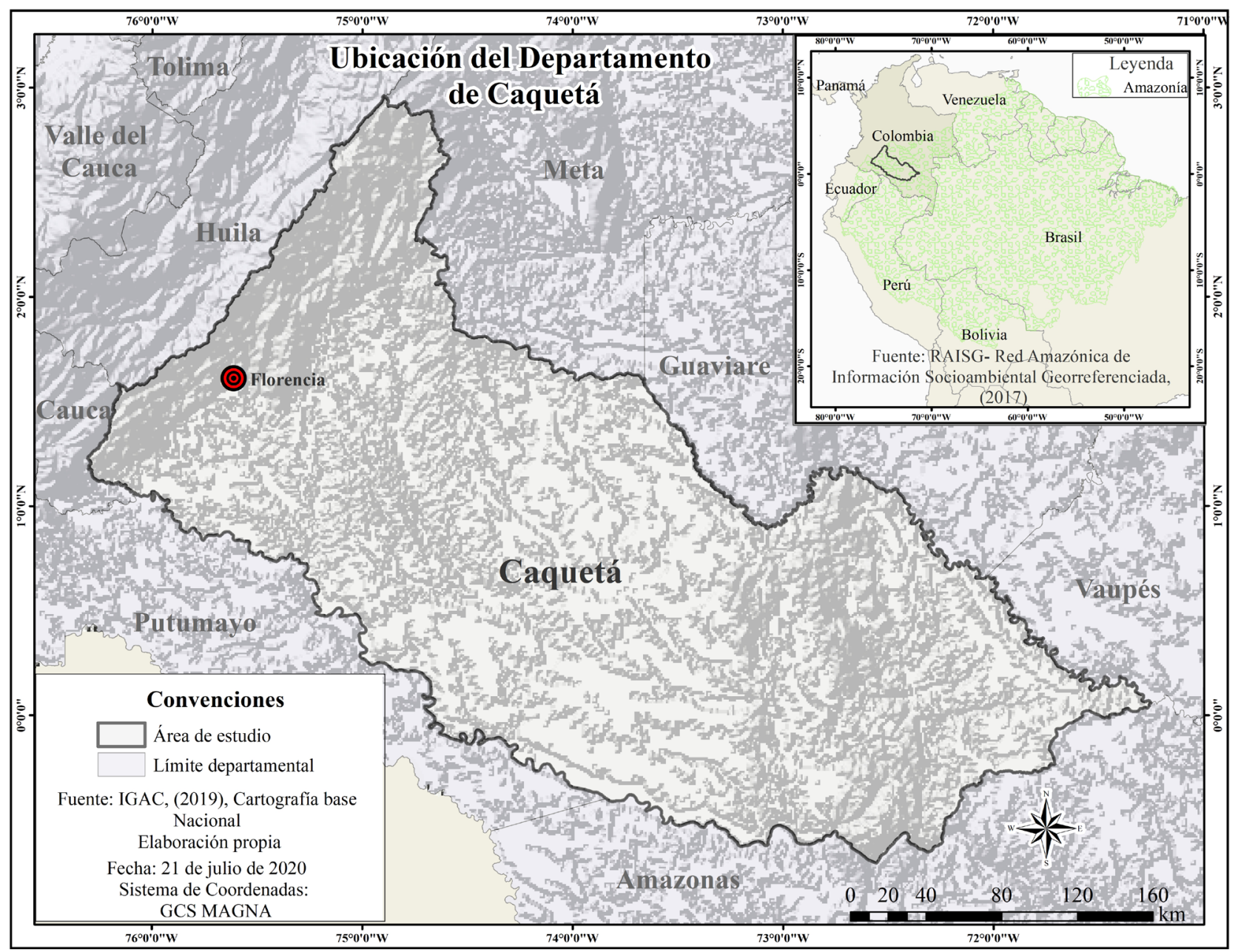

FIGURA 1. Área de estudio

FUENTE: elaboración propia.

\begin{tabular}{|c|cccccccc|c|}
\hline TABLA 1. Escala de costos & & & & \\
\hline Escala & 1 & 2 & 3 & 4 & 5 & 6 & 7 & 8 & 9 \\
\hline $\begin{array}{c}\text { característica } \\
\text { de los flujos }\end{array}$ & Alto movimiento & & $\begin{array}{c}\text { Movimiento } \\
\text { moderado }\end{array}$ & $\begin{array}{c}\text { Movimiento } \\
\text { con dificultad }\end{array}$ & Movimiento restringido \\
\hline
\end{tabular}

FUENTE: elaboración propia con base en Beier et al. (2009).

\subsection{Sistemas planetarios}

A continuación, se especifica la metodología para la asignación de costos para cada uno de los sistemas. Es importante mencionar que los elementos seleccionados para cada uno están condicionados por la disponibilidad de la información geográfica en cuanto a escala y temporalidad.

\subsubsection{Sistema atmosférico}

La precipitación se consideró como el elemento influyente dentro del sistema atmosférico, dado que esta favorece el equilibrio del agua tanto en sistemas superficiales como subterráneos. Altas precipitaciones compensan el consumo elevado de agua por la vegetación densa, dada su tasa de transpiración (Shaxson 
\& Barber, 2005). Se infiltra y percola alimentando las aguas subterráneas, corre como flujo superficial y es aprovechada por las plantas; favorece el flujo de nutrientes, microorganismos y materia orgánica en el suelo o simplemente satura la superficie (Shaxson \& Barber, 2005). La información utilizada corresponde al promedio multianual 1981-2010 (IDEAM, 2014) y los costos se asignaron dependiendo de la magnitud en milímetros de precipitación.

\subsubsection{Sistema hidrosférico}

La dinámica hidrológica contribuye a los fluidos vitales del paisaje (Etter, 1991). Por ejemplo, los ríos y quebradas son un sistema de circulación lineal per se con el propósito de dar sustento en todo su recorrido a la vida en sus diferentes formas (Campoblanco \& Gomero, 2000); las lagunas y pantanos son primordiales en el movimiento de especies, de nutrientes, en la regulación hídrica, climática y la calidad del aire (Borja et al., 2012).

Los costos fueron asignados dependiendo de las condiciones registradas en literatura secundaria sobre los cuerpos de agua, consignados en la cartografía base del departamento (Instituto Geográfico Agustín Codazzi [IGAC], 2018). Los valores dentro del primer intervalo (1-3) corresponden a los cuerpos de agua que no registran grandes perturbaciones; por el contrario, los costos de 5 y 6 se asignaron dependiendo del nivel de perturbación antrópica: el mayor costo (6) para cuerpos con presencia de mercurio, consecuencia de la explotación de oro (Salazar et al., 2019), y un costo de 5 a los cuerpos de agua cercanos o expuestos a la contaminación por aguas residuales.

En cuanto a la dinámica hidrogeológica, se tomaron las zonas potenciales de recarga de aguas subterráneas, clasificadas en alta, moderada, baja y muy baja potencialidad (IDEAM, 2019b), representadas a escala 1:1.000.000, con lo cual se rompe el parámetro de escala estimada para este estudio; sin embargo, se consideró útil ya que en términos hidrogeológicos se abarcan flujos hídricos a gran escala y la metodología que desarrolló el IDEAM (2010) tuvo en cuenta la variabilidad geográfica y geológica del país, indicando que todos los sistemas de flujo están contenidos dentro de escalas regionales, en este caso, el área que abarca la Amazonía colombiana (Thót, 1963).

Considerando de manera sistémica cuán influyente es cada zona según su potencialidad de recarga en el conjunto de flujos subterráneos, se asignaron los costos: a las zonas con mayor potencial de recarga les corresponde el menor costo del intervalo 1-3 por ser zonas de protección especial, ya que la Ley 99 de 1993 considera que "Las zonas de páramos, subpáramos, los nacimientos de agua y las zonas de recarga de acuíferos serán objeto de protección especial", y para las zonas de potencialidad moderada, baja y muy baja el costo aumenta conforme disminuye su potencialidad.

\subsubsection{Sistema geosférico}

Desde el componente edáfico fueron asignados costos por la presencia de los siguientes componentes en cada tipo de suelo: materia orgánica, minerales, microorganismos, aire y agua, puesto que la dinámica e interacción entre cada uno determina su funcionalidad (Shaxson \& Barber, 2005), con costos bajos si interactúan todos los componentes y costos altos si carecen de ellos.

Se analizaron las características de los suelos del departamento (IGAC, 2014) agrupados por el nivel de suborden según la clasificación del Departamento de Agricultura de los Estados (USDA, por su sigla en inglés) en Soil Survey Staff (2014) y se obtuvieron 12 diferentes subórdenes en los órdenes inceptisoles, entisoles, alfisoles, andisoles, ultisoles y oxisoles; además se identificaron tres suelos especiales asociados a centros urbanos, cuerpos de agua y afloramientos rocosos.

Respecto a los suelos especiales, los centros urbanos son catalogados como antrosoles debido a su fuerte transformación física y química; se trata de suelos 
compactos que carecen de poros, lo cual dificulta sus flujos (Organización de las Naciones Unidas para la Alimentación y la Agricultura [FAO], 2008). Los de los cuerpos de agua son denominados entisoles de cuerpos de agua por su relación con materiales aluviónicos, sin embargo, es importante aclarar que se considera que no existe suelo si la zona está cubierta en forma permanente por agua a 2,5 m de profundidad, lo cual impide por completo el desarrollo de vegetación (Soil Survey Staff, 2014). Por último, se encuentra el regolito que corresponde a partículas de rocas que no constituyen un suelo formado (Montoya-Rojas, 2018). Además, se tuvieron en cuenta los suelos con prioridad de conservación debido a su alta susceptibilidad a la erosión o su importancia por estar asociados a ecosistemas estratégicos como los páramos (Palacio, 2015).

\subsubsection{Sistema biosférico}

En este sistema, los elementos que condicionan el comportamiento de flujos son el tipo de vías y la cubierta biofísica. La cobertura está relacionada con organismos tanto animales como vegetales, ya que esta hace parte del entorno de las especies y particularmente de las características que determinan dónde estas se encuentran o no (Bamford \& Calver, 2014). Una estrategia para determinar los tamaños de los bloques de hábitat en los que se realizan actividades de conservación es la selección de un número pequeño de especies denominadas "especies focales", suponiendo que al usar los requisitos espaciales de estas en zonas suficientemente grandes e interconectadas cumplirían con los requisitos de las demás especies que allí habitan (Dinerstein et al., 2000, citado en Kattan et al., 2008). Para este sistema se utilizó esta metodología tomando tres especies focales según Kattan et al. (2008), quienes mencionan que se deben considerar especies emblemáticas de la región, con interés para conservación, que cumplan características ecológicas contrastantes y de las que se tenga información disponible. Además de esto, se verificó que estas especies estuviesen presentes en la mayoría de las áreas protegidas del departamento, ya que al contener áreas protegidas tanto en los Andes como en la Amazonía se garantizaba la distribución de las especies en las dos regiones. Así pues, las especies focales seleccionadas fueron el jaguar (Panthera onca), el mono churuco o mono lanudo gris (Lagothrix lagotricha lugens) y el águila arpía (Harpia harpyja).

Para la matriz de costo se relacionaron las coberturas que pudiesen restringir o no el movimiento fluido de las especies focales. Para ello, se tomaron las coberturas del Sistema de Monitoreo de las Coberturas de la Tierra de la Amazonía Colombiana (Instituto Amazónico de Investigaciones Científicas SINCHI, 2018), que están basadas en la metodología Corine Land Cover para Colombia; en este caso, se presentaron 45 coberturas. Cada cobertura fue calificada con un costo por especie focal, sin embargo, posteriormente se representó una matriz con la ponderación de los valores asignados. Se clasificaron en el intervalo de mayor costo (8-10) aquellas coberturas evitadas por las especies. Las coberturas que para cada especie representaran un lugar utilizable, pero no adecuado para su reproducción, fueron calificadas en el intervalo 6-7, mientras que se calificaron entre 4-5 aquellas que permiten una reproducción marginal y de 1-3 las coberturas que cumplen con las condiciones del hábitat reproductivo preferido por la especie (Beier et al., 2009).

Junto con las coberturas también se tuvieron en cuenta las vías, ya que estas tienen un impacto negativo sobre la fauna silvestre porque causan fragmentación, modificación de hábitat, cambio en los patrones reproductivos y mortalidad en la fauna que por allí transite (De La Ossa et al., 2015). Las vías tomadas de la cartografía base del departamento están clasificadas según su amplitud, el material con el que están construidas y el volumen de tráfico que circula por ellas (IGAC, 2010). Así pues, se calificaron según el peligro que estas representan para las especies: aquellas con mayor volumen de tráfico y revestidas de materiales 
que permiten la circulación a gran velocidad fueron calificadas dentro del intervalo más alto de la escala, mientras que aquellas que solo permiten el tráfico de vehículos de tracción animal o personas y sin revestimiento fueron contempladas en el siguiente intervalo.

\subsubsection{Sistema antroposférico}

En la matriz de costo de este sistema se tuvo en cuenta al ser humano como transformador de la naturaleza (Zubelzu \& Allende, 2015) y se tomó lo propuesto por Montoya-Rojas (2018) para analizar este sistema desde tres componentes, agrosistema, antroposistema y tecnosistema, para posteriormente representarlos en una única matriz.

Agrosistema: definido por Montoya-Rojas (2018) como aquel en el que se tiene en cuenta el servicio de abastecimiento, en especial de alimentos y materias primas. Mata Olmo et al. (2009, citado en Zubelzu \& Allende, 2015) indican que el paisaje visto por el ser humano es concebido como sustento de actividades productivas. Por esto, se tomó la capa de coberturas del 2018 del departamento, ya mencionada en el sistema anterior, pero ahora con énfasis en las coberturas intervenidas por el ser humano para dichas actividades, como los territorios agrícolas y áreas seminaturales en las que, según la Corporación para el Desarrollo Sostenible del Sur de la Amazonia (Corpoamazonia, 2012), se llevan a cabo actividades como ganadería y agricultura. También se consideró la extracción de hidrocarburos y la pesca, ya que esta última es una las actividades productivas más importantes de la región amazónica.

Cada una de las actividades mencionadas genera demanda del suelo y por ello la Unidad de Planificación Rural Agropecuaria (UPRA, 2014) tiene en cuenta la vocación de este. Basándose en esto, se relacionaron únicamente las coberturas intervenidas por el ser humano con la vocación del suelo, información que fue obtenida de los Mapas de Clasificación de las Tierras por su Vocación de Uso a escala 1:100.000 elaborados por el IGAC (2017), donde se especifica que el objetivo principal de la vocación es la determinación del uso más apropiado que puede soportar cada suelo, buscando una producción que no deteriore los recursos naturales. Este insumo contiene un atributo referente al uso principal recomendado desde el punto de vista del desarrollo sostenible y fue usado para realizar la comparación (IGAC, 2013). La asignación de costos se realizó de la siguiente manera: costo de 1 para aquellas coberturas que por su actividad coincidieran con la vocación del suelo y luego, para aquellas coberturas que no tuviesen coincidencia, se les calificó según la categorización por niveles del estado de conservación de los suelos propuesta por el Instituto SINCHi para los suelos de la Amazonía (Peña-Venegas \& Cardona, 2010).

Ahora bien, para adecuar cada uno de los niveles a la escala manejada por la metodología de Beier et al. (2009) fueron calificadas las coberturas del nivel 1 dentro del intervalo de 2-3, luego las de nivel 2 en el intervalo de 4-5, las de nivel 3 de 6-7 y aquellas que se encontraran en el nivel 4 y 5 se les dio un costo dentro del intervalo de 8-10.

Antroposistema: este componente es definido como el suelo transformado por el ser humano para la construcción de infraestructura que satisface sus necesidades básicas como salud, educación, vivienda, empresas y vías (Montoya-Rojas, 2018). En el área de estudio se identificaron como parte de este los aeropuertos, las áreas urbanas, las vías y la red de ríos navegables. Esta infraestructura fue calificada según su contribución en la interacción de la población; Rozas y Figueroa (2006) mencionan que la representación física del concepto abstracto de conectividad es la estructura conformada por redes de corredores que sirven para movilizar bienes, servicios y personas entre distintos puntos del territorio.

En primer lugar, el transporte aéreo contribuye a la conexión y la inclusión social porque promueve la conectividad interregional y facilita el comercio y el intercambio de conocimientos e ideas (Rozas \& 
Figueroa, 2006), por esta razón los aeropuertos del área del departamento que permiten un alto movimiento de flujos representan un costo 1 .

Otro elemento que se evaluó son las zonas urbanas de los municipios, que conforman conjuntos de estructuras agrupadas y delimitadas por calles o carreras. Estas áreas cuentan por lo general con dotación de servicios esenciales como acueducto, alcantarillado, electricidad, gas, colegios y hospitales (DANE, 2009). Al ser puntos de concentración de estos servicios para el ser humano, se les asignó igualmente un costo de 1.

Respecto a las vías, las condiciones de estas determinan los tiempos de viaje, los costos productivos, la accesibilidad a mercados y la generación de bienestar social (Miranda, 2016). A mayor diversidad vial entre vías de tipo 1 a 3, mejor será el bienestar socioeconómico (Durango, 2016), por esta razón las vías tipo 1 y 3 representan un costo de 1. Luego las demás fueron calificadas según el volumen de tráfico que permiten y la calidad de su revestimiento: las vías tipo 4 y 5 se encuentran en el intervalo de costo de 4-5 debido a que permiten un movimiento moderado y las vías tipo 6 y 7 cuentan con un costo de 6 ya que por ellas se dificulta el movimiento.

A pesar de que los ríos son elementos naturales, han pasado a ser entendidos como parte del ensamblaje dentro de la infraestructura de transporte (Serje \& Ardila, 2017), por lo cual se tomaron en cuenta los ríos pertenecientes a la red primaria y segundaria de navegación. En la red primaria está el río Caquetá y en la secundaria los ríos Caguán, Orteguaza, Apaporis y Yarí, según el Ministerio de Transporte (2010). Corpoamazonia (2011) menciona la importancia de esta red fluvial a través de la cual se establece comunicación entre las zonas rurales más apartadas de la concentración urbana del departamento y por esta razón fueron calificados dentro del intervalo 1-3 debido a que por medio de ellos se facilita el movimiento.

Tecnosistema: en este componente se incluyen las zonas que cuentan con alguna figura legal de conservación o manejo especial (Montoya-Rojas, 2018). Bajo esa definición se tomaron las diferentes áreas protegidas del Sistema Nacional de Áreas Protegidas de Colombia (SINAP) y otras áreas con figuras legales de conservación, como resguardos indígenas y zonas de reserva campesina.

En cuanto a los resguardos indígenas y las zonas de reserva campesina, el Instituto de Investigación de Recursos Biológicos Alexander von Humboldt (IAVH) y la Fundación Natura (2019) indicaron que estos territorios son de gran importancia en términos de biodiversidad, servicios ecosistémicos y valores culturales debido a las prácticas de estos grupos a raíz de las formas de gobernanza comunitarias.

Teniendo en cuenta lo anterior, se dio un costo a cada una de estas áreas representadas a partir del nivel de conservación y el manejo especial de recursos naturales a través de las figuras legales que dichas áreas representan. En este caso, se asignaron valores en el intervalo de 1-3, puesto que todas las áreas presentan un manejo especial y preservan alguna parte del sistema natural, unas en mayor grado que otras. Las áreas calificadas con 2 son aquellas que permiten el uso de los recursos naturales dentro de estas, pero sin una población beneficiaria específica. Esto podría dar lugar a conflictos, como en el caso de las reservas forestales protectoras y los parques naturales regionales que se encuentran coordinados por dos Corporaciones Autónomas Regionales distintas, con excepción del Parque Natural Regional Miraflores Picachos (Bonilla \& Higuera, 2016). La áreas calificadas con 1 tenían un manejo especial tanto de forma institucional como de la población que las habita, dadas las relaciones de gobernanza donde las reglas de uso y gestión se producen en deliberación entre actores heterogéneos (Palacio, 2015), contribuyendo de forma significativa el cumplimiento de los objetivos de conservación planteados para cada área protegida (Cortes, 2018). Aquellas áreas sin figura legal de conservación, pero con cobertura vegetal no modificada, representaron un costo de 3. 


\subsubsection{Superficie de costo a partir de los sistemas}

Finalmente, se realizó la superficie de costo con la herramienta Weighted Overlay de ArcGIS a partir del procesamiento conjunto de las cinco matrices de costo resultantes de cada sistema mencionado, donde cada sistema tiene la misma importancia dentro de la superficie, contribuye en un $20 \%$ al procesamiento y como resultado se obtiene la representación del costo de la propagación conjunta de los flujos considerados dentro del paisaje del departamento de Caquetá.

\section{Resultados}

Los resultados representados en la Figura 2 (a-e) son las matrices de costo, un conjunto de mapas que tras el análisis y la asignación de costos a los elementos de cada sistema planetario muestra una aproximación de los lugares y la respuesta de los flujos frente a la configuración del paisaje, con los respectivos porcentajes que ocupan en el área de estudio.

Para el sistema atmosférico se identificaron precipitaciones entre 1.000 y $4.000 \mathrm{~mm}$ anuales, donde la mejor valoración en la escala de costo es para las zonas con mayor magnitud, ya que estas magnitudes en la región favorecen el alto movimiento de flujos, ya sea transitando por los suelos, compensando el consumo elevado de la vegetación o contribuyendo a la recarga de acuíferos. Alrededor del 64\% del departamento presenta un costo de 1 , mientras que los costos de 2 y 3 corresponden al 36\% restante.

En el sistema hidrosférico se reunieron los cuerpos de agua superficiales y las zonas potenciales de recarga y se obtuvo como resultado una matriz conjunta con costos entre 1 y 6 . Se aprecia cómo las zonas con el menor costo están en inmediaciones de la serranías de Chiribiquete y de Araracuara, donde el costo 3 tiene la mayor cobertura, con casi el 53\% del área de estudio, mientras que costos más altos (5 y 6) corresponden a los flujos relacionados con los cuerpos de agua contaminados con aguas residuales o metales pesados; sin embargo, estos últimos ocupan menos del 1\% del área total del departamento, lo cual da a entender que en términos del sistema hidrosférico se tiene un alto movimiento de flujos.

El tercer sistema, el geosférico, dio como resultado que menos del 0,1\% del área de estudio corresponde a antrosoles, como suelos asociados a los centros poblados del departamento. En su mayoría, los ríos principales y algunas rocas o regolitos presentan costos de 5 ya que por sus características no permiten el flujo de la totalidad de los componentes del suelo, sin embargo, en términos generales, el área de estudio, en un $76 \%$, presenta costos dentro del primer intervalo (1-3), denotando así un alto movimiento de los flujos asociados a los suelos.

El sistema biosférico junto con el sistema antroposférico, dada su dinámica, son los que contienen valores dentro de los cuatro rangos de la escala. En el biosférico, las zonas con costos de 8 a 10 cubren el $21 \%$ del departamento y las que presentan costos de 4 a 7 abarcan un poco menos del 10\%. Sin embargo, aun cuando resalta el área donde la propagación de flujos, en este caso, de las especies focales, se ve limitada, en la mayor parte $(69 \%)$ del departamento estas no se ve afectadas.

El águila arpía es sensible a la contaminación y a cambios en la calidad del hábitat (Piana, 2007), ya que prefieren principalmente bosques primarios no inundables o lugares con árboles altos para anidar, sin embargo, también pueden hacerlo cerca a zonas intervenidas de forma no mecanizada por el ser humano. Por el contrario, los lugares con extracciones forestales y explotación de palmeras con fines comerciales y cercanos a vías principales, aunque son transitados por el ave, no son sus áreas preferidas, ya que los nidos que allí construyen son en su mayoría abandonados por el ave (Piana, 2007).

En cuanto al jaguar, Payán y Soto (2012) describen, como amenazas para la especie, la cacería, 
particularmente por parte de ganaderos, la fragmentación de la población por las vías, el avance de la frontera agrícola y la minería (Payán et al., 2015). Se encuentra muy activo durante el día o la noche en zonas de baja perturbación antrópica, sin embargo, se ha observado la especie en cercanía a zonas de plantaciones de palmas de aceite y bosques secundarios $(\mathrm{Pa}$ yán et al., 2015). Payán y Soto (2012) describen que esta especie frecuenta ríos y depósitos de agua dulce y que las poblaciones de jaguar habitan bosques y sabanas tropicales, así como bosques de galería asociados a ríos, ciénagas y playones, y también señalan que las coberturas en las que se evidencian bosques fragmentados están relacionadas con otros cambios ecológicos como la caza, las quemas y la tala, además de que el tamaño de los parches empieza a limitar su supervivencia y afirman que este es el mamífero que primero desaparece con el avance de la frontera agrícola.

En cuanto al mono churuco, este evita las coberturas donde no hay bosque puesto que suele habitar en árboles altos y ocasionalmente baja al suelo. Ya que es improbable que pueda subsistir en otro hábitat diferente (National Research Council, 1976), es importante recalcar que esta especie tiene una función primordial en la dispersión de semillas por ser un animal frugívoro, pero actualmente se ve amenazado por la pérdida de su hábitat debido a la cacería y principalmente a la deforestación (Stevenson \& Guzmán-Caro, 2014).

Por último, tenemos el sistema antroposférico: como resultado de la agrupación del agrosistema, el antroposistema y el tecnosistema se obtiene una matriz donde el menor costo (1) representa el 52,4\% del departamento, que corresponde principalmente a las áreas protegidas y los diferentes resguardos indígenas, seguido de costos 2 y 3 con el 35,6\%, lo cual demuestra que casi el $88 \%$ de este paisaje favorece el movimiento alto de flujos.

Posterior a esto, se presenta la superficie de costo como resultado del estudio efectuado desde la ecología del paisaje, ya que resulta de la ponderación de las matrices anteriores, es decir, es la que reúne de manera sistémica la configuración del paisaje y la respuesta de los flujos a esta configuración (Figura 2f). Aun cuando en las matrices se obtienen costos altos, esta superficie cuenta con costos de 1 a 6 debido a la ponderación realizada, donde el intervalo que determina el alto movimiento de flujos cubre alrededor del 83\% del área y solo el 16\% corresponde a costos de movimientos moderados y con dificultad.

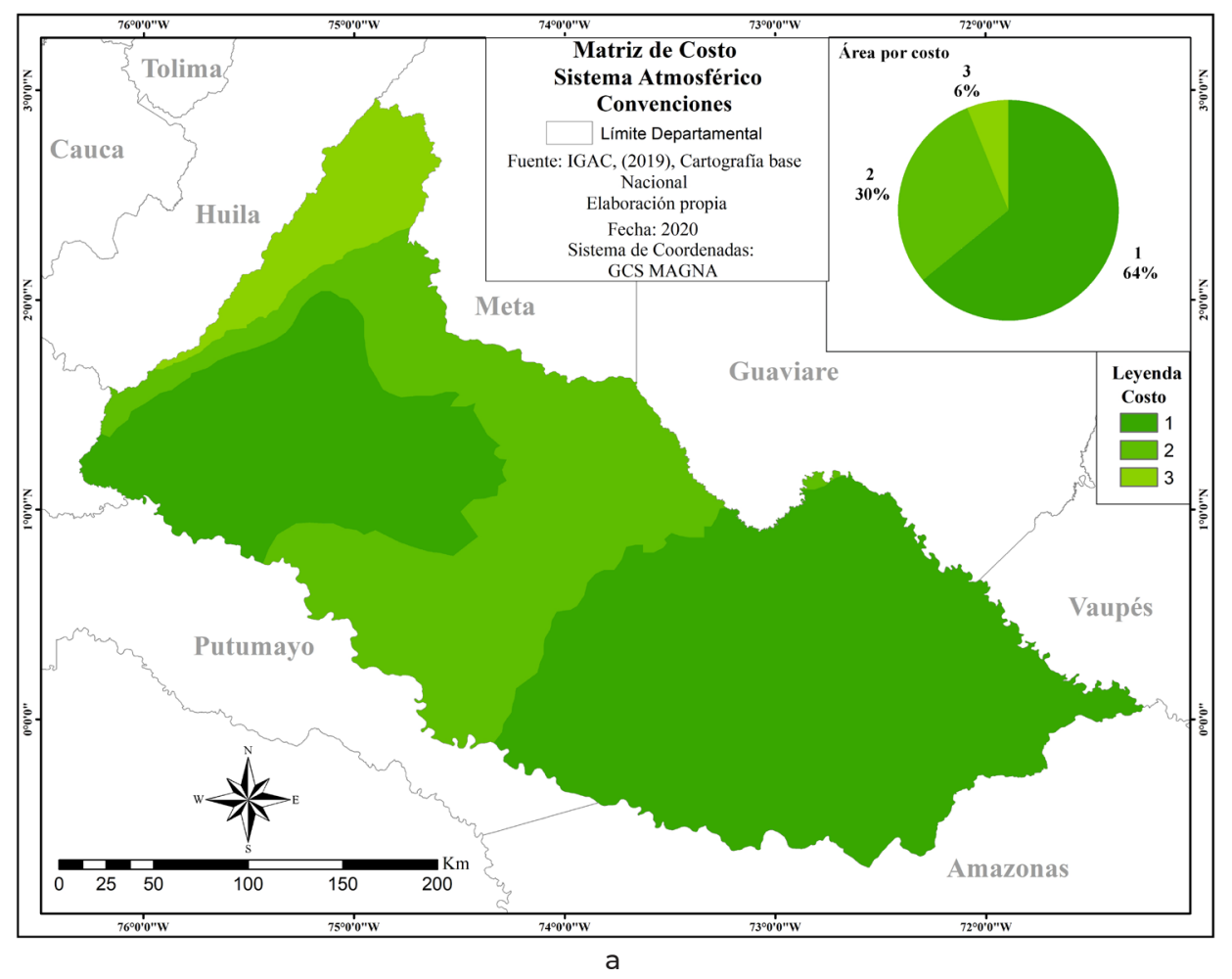




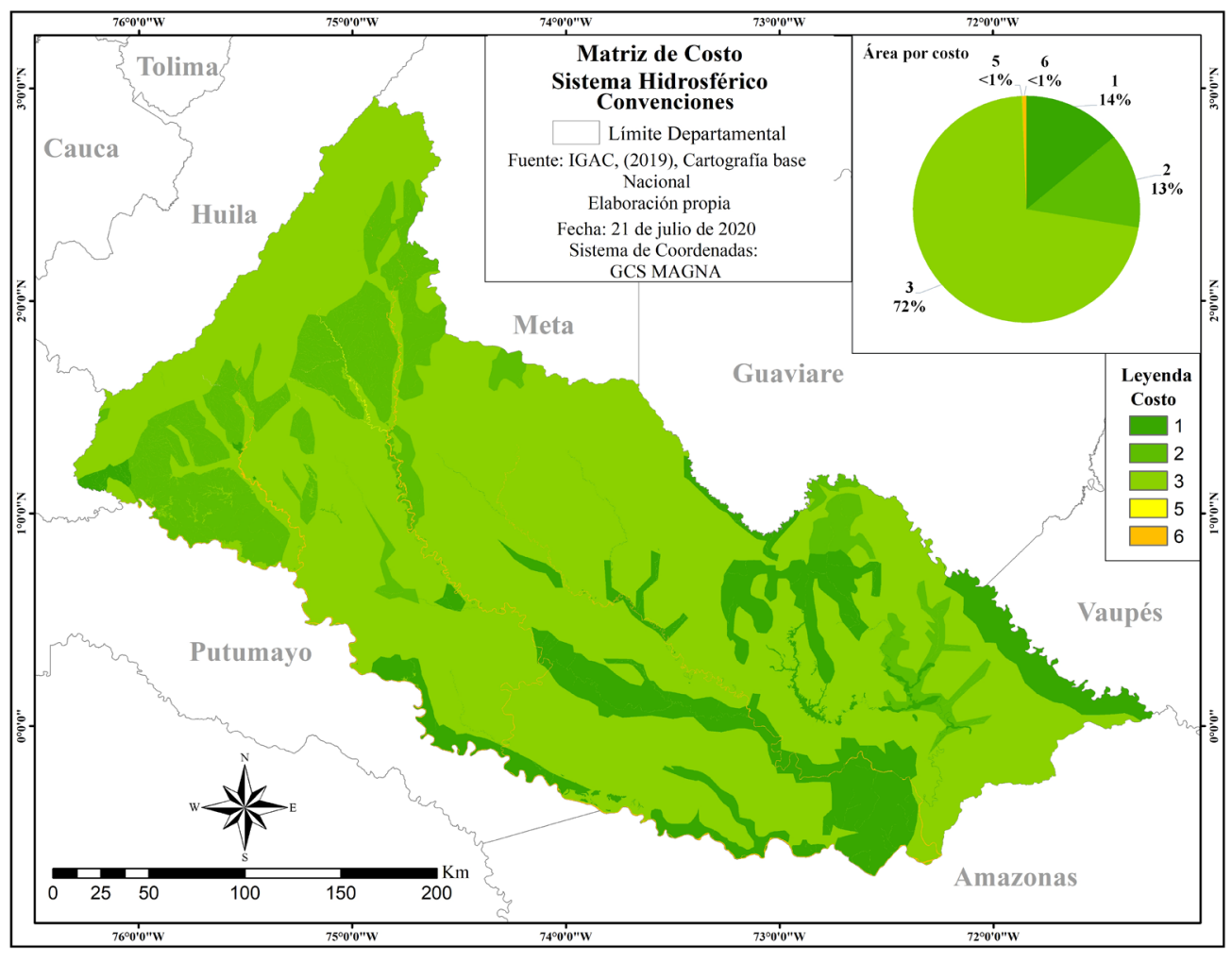

b

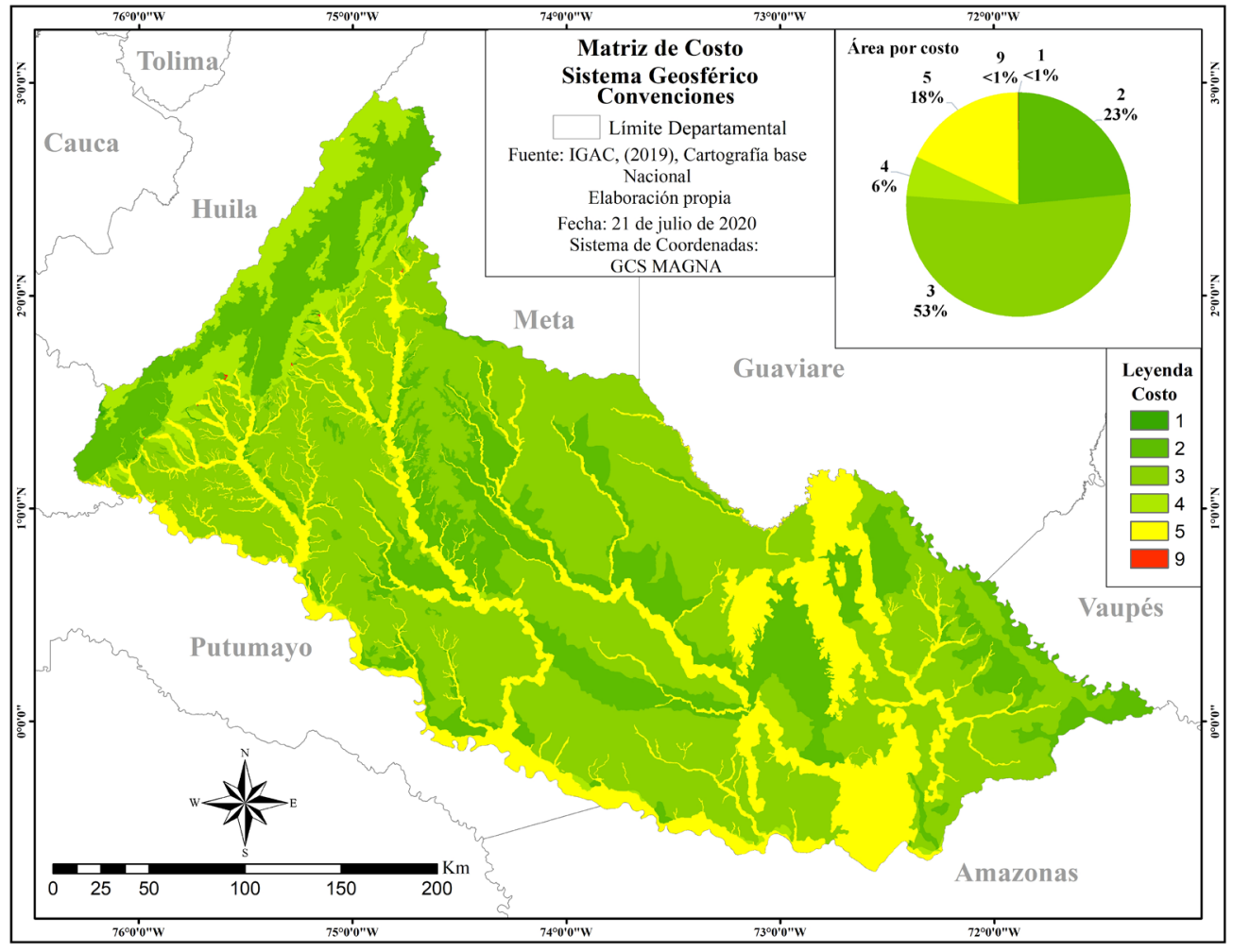

C 

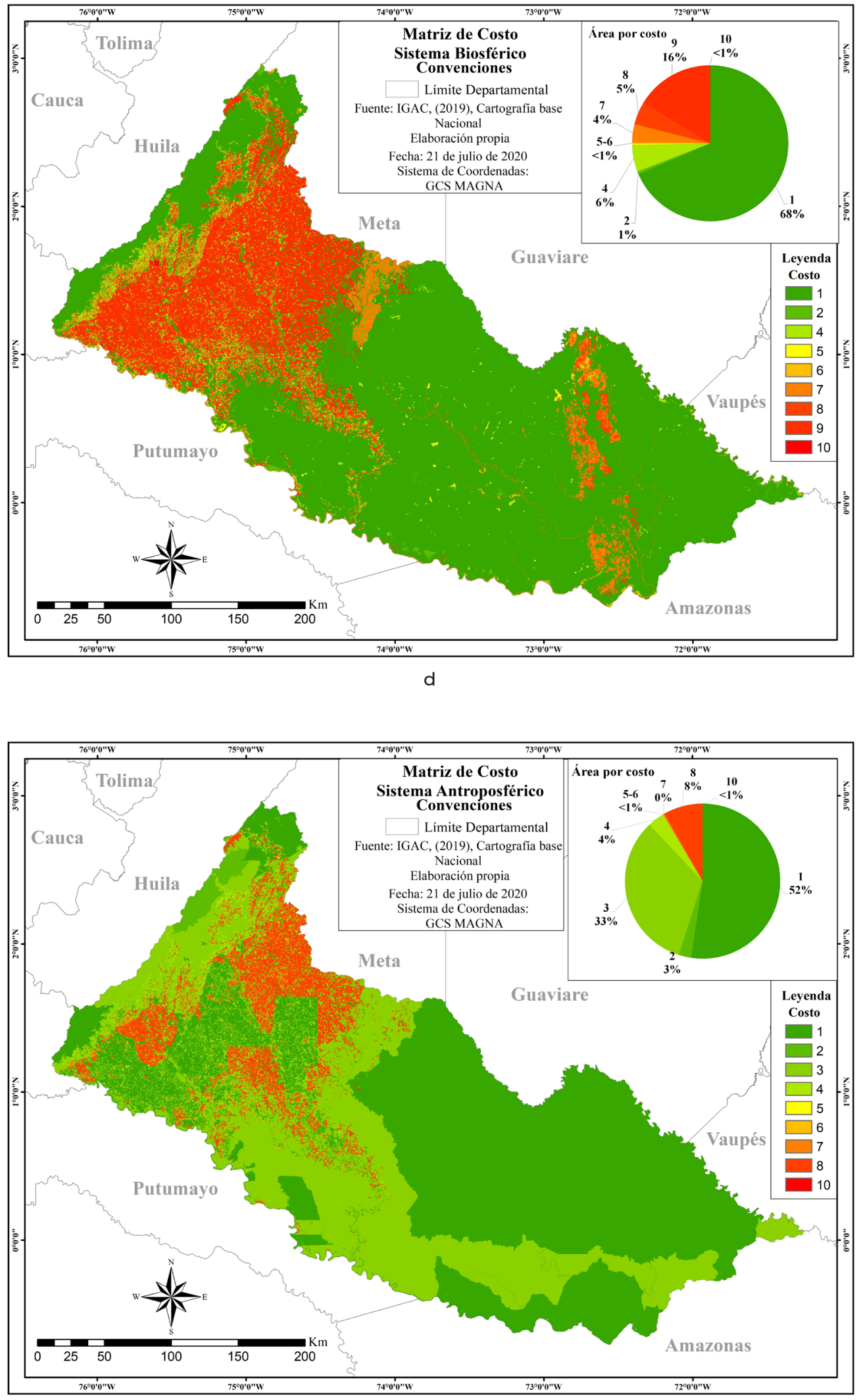

e 


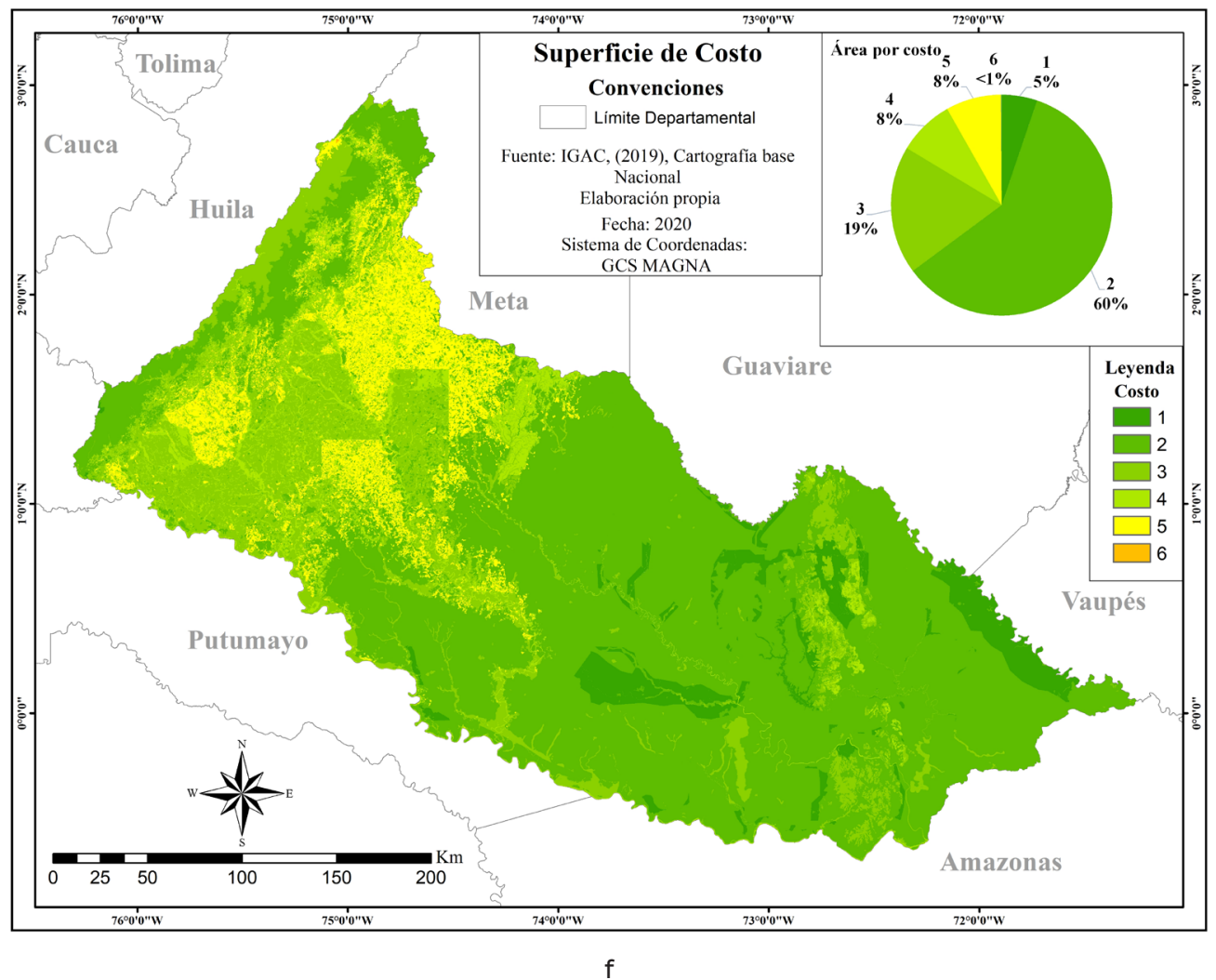

FIGURA 2. Matrices y superficie de costo: a) sistema atmosférico; b) sistema hidrosférico; c) sistema geosférico; d) sistema biosférico; e) sistema antroposférico; f) superficie de costo FUENTE: elaboración propia a partir de datos cartográficos de IDEAM (2014, 2019b), IGAC (2017, 2018) e Instituto SINCHI (2018).

\section{Discusión}

Para analizar el estado de la conectividad entre los Andes y la Amazonía desde la ecología del paisaje en el departamento de Caquetá se debe entender la interrelación que existe entre los diferentes elementos que lo componen. Inicialmente, cuando la cordillera de los Andes emerge, provoca la reconfiguración de los elementos del paisaje y da como resultado lo que hoy se conoce como la Amazonía, que genera la dependencia de estas dos regiones para cumplir con sus funciones ecológicas. Por ejemplo, los vientos que atraviesan la selva amazónica transportan consigo la humedad que allí recogen, la llevan hasta la cordillera y provocan que la precipitación de esta región sea alta y que el departamento de Caquetá cuente con una riqueza hídrica compuesta por diferentes humedales, lagunas, pantanos, quebradas y grandes ríos como el
Caguán, el Orteguaza y el Caquetá, que nacen en la cordillera, o el Yarí y el Apaporis, que nacen en la planicie amazónica. Esta riqueza permite que entre la selva y la montaña existan distintos ecosistemas que producen la diversidad de fauna y flora que caracteriza estas regiones.

La erosión de los Andes, donde hay suelos nuevos ricos en minerales, contribuye al enriquecimiento de suelos en las zonas bajas del departamento, especialmente alrededor de grandes ríos. El 75\% de los suelos del área de estudio son antiguos y pertenecen a los órdenes oxisol y ultisol, que contienen minerales y micronutrientes que soportan ecosistemas selváticos y de riqueza endémica, lo cual permite la interacción paisajística en zonas tropicales y subtropicales principalmente (Gutiérrez-Alfonso, 2016; Montoya-Rojas \& Rivera-Marroquín, 2021), si bien son susceptibles a degradarse, es decir, a la pérdida de sus características 
propias por actividades antrópicas, especialmente por prácticas ganaderas que lo compactan (Peña-Venegas \& Cardona, 2010). Para las aguas subterráneas, las zonas de recarga que se encuentran en la parte baja, en inmediaciones de la serranía de Chiribiquete, dependen de las características propias de la geología de esta zona que permite infiltraciones y de la precipitación que discurre desde la cordillera (IDEAM, 2019b).

Llama la atención que en la selva amazónica hacia la serranía de Chiribiquete, donde se encuentra la mayor área que favorece al flujo de especies, también presente fragmentos con costos altos debido a las formaciones rocosas, las cuales son de poca preferencia por las especies focales; sin embargo, por las características del área circundante, es posible encontrar allí varios ejemplares de estas especies.

La mayor parte de la población del departamento se encuentra en las zonas urbanas que por lo general cuentan con dotación de servicios esenciales y están conectadas por vías de tipo 6 y 7 en su mayoría. Del $100 \%$ de la red vial con la que cuenta el departamento, el $76 \%$ es de este tipo y solo un $2 \%$ son vías de tipo 1 , lo cual señala que hay dificultad en la comunicación entre zonas urbanas.

Por otro lado, como figuras legales de conservación o manejo especial dentro del departamento, se encontró una zona de reserva campesina en la cual se llevan a cabo prácticas de conservación y uso sostenible de la biodiversidad, lo cual se evidencia en las coberturas dentro del área. El sur del departamento se encuentra protegido bajo la figura de 24 resguardos indígenas, el oeste por tres parques naturales nacionales, tres reservas naturales de la sociedad civil y un parque natural regional y, por último, al este, ocupando un 36\% del departamento, se encuentra el Parque Natural Nacional Serranía de Chiribiquete, el único PNN del departamento que se encuentra en su totalidad sobre la región amazónica. Es importante mencionar que estas áreas protegidas son compartidas con los departamentos del Guaviare, Meta, Huila y Cauca.
Por un lado, los resguardos indígenas con una titulación de la tierra colectiva, por medio del Plan de Vida que los acobija, identifican áreas de conservación, sitios sagrados y corredores interculturales, dado que las tradiciones y la cosmovisión crean un estrecho vínculo con el territorio y la tierra, la cual es considerada como Madre (Cortes, 2018). Por el otro lado, en el departamento se encuentra una zona de reserva campesina que cuenta con titulación de la tierra dada a una organización o asociación campesina, por medio de una propuesta territorial propia, con el desarrollo de estrategias y prácticas de conservación y uso sostenible de la biodiversidad (Agencia Nacional de Tierras [ANT], 2017), lo cual muestra la capacidad que tienen las comunidades de conservar, gestionar y beneficiarse del ecosistema (Tocancipá Falla \& Ramírez Castrillón, 2018).

Los costos más altos ocupan alrededor del 8,5\% del área y están relacionados con las coberturas que no coinciden con la vocación del suelo. El parche de mayor tamaño se encuentra en el municipio de San Vicente del Caguán. Peña-Venegas y Cardona (2010) señalan que la actividad ganadera allí, traducida en pastos limpios, se debe a la expansión de esta práctica desde las praderas del departamento del Meta.

Según Cortes (2018), la conservación solo es posible si los actores sociales que hacen uso de los recursos naturales están incluidos en los espacios de participación dentro del área protegida. De esta forma se sustenta la importancia de aquellos territorios indígenas dentro de los PNN, ya que al promover y consolidar procesos de participación social se llega a la coordinación para la conservación, dando un reconocimiento a aquellas comunidades que han vivido el territorio (Van der Hammen et al., 2015, citado en Cortes, 2018).

\section{Conclusiones}

Tanto el sistema atmosférico como el hidrosférico demostraron que las altas precipitaciones y la dinámica hidrológica favorecen el movimiento del agua desde que esta precipita, filtra, discurre y alimenta las zonas 
de recarga subterráneas, con una dinámica continua y sin mayor perturbación.

Las características del buen drenaje de los suelos más antiguos (oxisoles y ultisoles), localizados en el piedemonte y en algunas zonas de montaña sobre los Andes, hicieron que en el 76,2\% del área se favorezca la propagación de flujos asociados al sistema geosférico. Fue posible identificar que la mayor perturbación al movimiento de flujos está en menos del 1\% del área de estudio asociada a los antrosoles, y con un 18\% y costo de 5 se asocian los cuerpos de agua y regolitos que por su ineficiencia en los procesos de drenaje adquieren esta calificación.

La mayor perturbación encontrada en el análisis está asociada a modificaciones antrópicas y su relación con el sistema biosférico, donde el 21\% del área total corresponde al intervalo más restrictivo para los flujos. Este porcentaje tiene relación con las coberturas transformadas por el ser humano que están concentradas casi en su totalidad sobre el piedemonte, donde se asienta la mayoría de la población. Esto hace que aun cuando el 69\% del área del departamento cumpla con las condiciones óptimas para las especies seleccionadas, su conectividad se vea fragmentada.

Para el sistema antroposférico, al relacionar el paisaje del departamento y las dinámicas que las actividades antrópicas representan, se observa una alta movilidad de flujos atribuida en gran medida al tecnosistema al considerar las áreas protegidas de nivel nacional y los resguardos indígenas, que en conjunto abarcan el 46\% del área del departamento; las zonas con mayor resistencia están relacionadas con las coberturas que no coinciden con la vocación del suelo. De manera general, al relacionar el análisis del conjunto de sistemas y desde la perspectiva de la ecología del paisaje, se demuestra que en la configuración del paisaje del departamento de Caquetá sus elementos están ordenados de tal manera que los flujos no presentan restricciones ni mayores dificultades para movilizarse y conectarse entre las regiones de los Andes y la Amazonía colombiana.

\section{Referencias}

Agencia Nacional de Tierras (ANT) (2017). ¿Qué son las Zonas de Reserva Campesina? http://consejoderedaccion.org/ webs/documentos/Respuesta \%20Agencia $\% 20 \mathrm{Na}$ cional $\% 20 \mathrm{de} \% 20$ Tierras.pdf

Balbi, M., Petit, E. J., Croci, S., Nabucet, J., Georges, R., Madec, L., \& Ernoult, A. (2019). Ecological relevance of least cost path analysis: An easy implementation method for landscape urban planning. Fournal of Environmental Management, 244, 61-68. doi.org/10.1016/j. jenvman.2019.04.124

Bamford, M. \& Calver, M. (2014). A precise definition of habitat is needed for effective conservation and communication. Australian Zoologist, 37(2), 245-247. doi. org/10.7882/AZ.2014.015

Beier, P., Majka, D., \& Newell, S. (2009). Uncertainty analysis of least-cost modeling for designing wildlife linkages. Ecological Applications, 19(8), 2067-2077. doi. org/10.1890/08-1898.1

Bonilla, L. \& Higuera, I. (2016). ¿Parques de papel? Áreas protegidas y deforestación en Colombia. Serie Documentos de Trabajo Sobre Economía Regional, n² 248. Centro de Estudios Económicos Regionales (CEER) del Banco de la República. doi.org/10.32468/dtseru.248

Borja, C., Florin, M., \& Camacho, A. (2012). Lagos y humedales de interior. En Ministerio de Medio Ambiente y Medio Rural y Marino (ed.), Evaluación de Ecosistemas del Milenio de España (pp. 1-63). https://www. researchgate.net/publication/264556756_Lagos_y_ humedales_de_interior

Campoblanco, H. \& Gomero, J., (2000). La importancia de los ríos en el entorno ambiental. Revista del Instituto de Investigación de la Facultad de Geología, Minas, Metalurgía y Ciencias Geográficas, 3(5), 57-63. https://revistasinvestigacion. unmsm.edu.pe/index.php/iigeo/article/view/2539

Ciro-Rodríguez, E. (2018). "Ni guerra que nos mate, ni paz que nos oprima": incursión petrolera y defensa del agua durante las negociaciones y firma de la paz en el sur de Colombia. Colombia Internacional, 93, 147-178. dx.doi.org/10.7440/colombiaint93.2018.06

Clerici, N., Salazar, C., Pardo-Díaz, C., Jiggins, C., Richardson, J., \& Linares, M. (2019). Peace in Colombia is a critical moment for Neotropical connectivity and conservation: Save the northern Andes-Amazon biodiversity bridge. Society for Conservation Biology, Conservation Letters, 12(1), 1-7. https://doi.org/10.1111/conl.12594 
Comisión Económica para América Latina y el Caribe (CEPAL) \& Patrimonio Natural, Fondo para la Biodiversidad y Áreas Protegidas (2013). Amazonia posible y sostenible. CEPAL. https://www.cepal.org/es/publicaciones/1506-amazonia-posible-sostenible

Corporación para el Desarrollo Sostenible del Sur de la Amazonia (Corpoamazonia) (2011). Caracterización ambiental, Plan Departamental de Agua Departamento de Caquetá. https://www.corpoamazonia.gov.co/files/Documento_Caquet $\% \mathrm{C} 3 \% \mathrm{~A} 1 . p d f$

Corporación para el Desarrollo Sostenible del Sur de la Amazonia (Corpoamazonia) (2012). Actividades Productivas de Caquetá. Corpoamazonia. https://www.corpoamazonia.gov.co/region/Jur_Actividades.htm

Correa-Ayram, C. (2017). La huella espacial humana en la conectividad del paisaje e identificación de áreas importantes para su conservación. En Global Online Seminar Series in Biodiversity Informatics. doi.org/10.13140/ RG.2.2.14541.03044

Cortes, A. (2018). Resguardos Indígenas en la conservación: territorios en traslape. [Tesis de especialización]. Universidad Externado de Colombia. https://bdigital.uexternado.edu.co/bitstream/001/682/1/DHA-spa-2018Resguardos_indigenas_en_la_conservacion_territorios_ en_traslape.pdf

De La Ossa, V., De La Ossa-Nadjar, O., \& Medina-Bohórquez, E. (2015). Atropellamiento de fauna silvestre. Revista Colombiana de Ciencia Animal - RECIA, 7(1), 109116. doi.org/10.24188/recia.v7.n1.2015.430

Departamento Administrativo Nacional de Estadística (DANE) (2009). Conceptos básicos. https://www.dane.gov. co/files/inf_geo/4Ge_ConceptosBasicos.pdf

Departamento Administrativo Nacional de Estadística (DANE) (2018). Censo Nacional de Población y Vivienda 2018, departamento de Caquetá. https://microdatos.dane.gov.co/index.php/catalog/643/datafile/ F11/V574

Durango, A. (2016). Relación entre infraestructura vialy desarrollo económico en los municipios de Antioquia: aplicación espacial. [Tesis de maestría]. Universidad EAFIT. https://repository.eafit.edu.co/bitstream/handle/10784/11897/ EdiliaAndrea_DurangoAgudelo_2016.pdf?sequen$\mathrm{ce}=2$ \&isAllowed $=\mathrm{y}$

Etter, A. (1991). Introducción a la ecología del paisaje: un marco de integración para los levantamientos ecológicos. Landscape Ecology, 32(3), 25-56. doi. org/10.13140/2.1.4464.5121
Gurrutxaga, M., \& Lozano, P. (2008). Ecología del paisaje. Un marco para el estudio integrado de la dinámica territorial y su incidencia en la vida silvestre. Estudios Geográficos, 69(265), 519-543. doi.org/10.3989/estgeogr.0427

Gutiérrez-Alfonso, K. (2016). Transformación del paisaje en el Departamento del Meta una zona de conflicto armado. [Tesis de pregrado]. Universidad de Ciencia Aplicadas y Ambientales - U.D.C.A. https://repository.udca. edu.co/handle/11158/1043

Instituto Amazónico de Investigaciones Científicas SINCHI (2018). Monitoreo de los bosques y otras coberturas de la Amazonia Colombiana, a escala 1:100.000. Instituto sINCHI. https://sinchi.org.co/files/publicaciones/publicaciones/pdf/MONITOREO \%20web.pdf

Instituto de Hidrológia Meteorología y Estudios Ambientales (IDEAM) (2010). Estudio Nacional del Agua. 2010. http://documentacion.ideam.gov.co/openbiblio/ bvirtual/021888/021888.htm

Instituto de Hidrología, Meteorología y Estudios Ambientales (IDEAM) (2014). Atlas de precipitación media total anual. Promedio multianual 1981-2010. http:/ / atlas.ideam.gov. co/visorAtlasClimatologico.html

Instituto de Hidrología, Meteorología y Estudios Ambientales (IDEAM) (2017). Resultados Monitoreo de la Deforestación 2017. http://documentacion.ideam.gov.co/openbiblio/bvirtual/023835/Resultados_Monitoreo_Deforestacion_2017.pdf

Instituto de Hidrología, Meteorología y Estudios Ambientales (IDEAm) (2018). Boletín de Detección Temprana de Deforestación, $\mathrm{n}^{\circ}$ 17. http://documentacion.ideam.gov. co/openbiblio/bvirtual/023856/17_Boletinat-D. pdf

Instituto de Hidrología, Meteorología y Estudios Ambientales (IDEAM) (2019a). Boletín de Detección Temprana de Deforestación, $\mathrm{n}^{\circ}$ 21. http://documentacion.ideam.gov. co/openbiblio/bvirtual/023884/023884.html

Instituto de Hidrología, Meteorología y Estudios Ambientales (IDEAm) (2019b). Estudio Nacional del Agua 2018. http://documentacion.ideam.gov.co/openbiblio/ bvirtual/023858/ENA_2018.pdf

Instituto de Investigación de Recursos Biológicos Alexander von Humboldt (IAVH) \& Fundación Natura (2019). Voces de la gestión territorial: estrategias complementarias para la conservación de la biodiversidad en Colombia. Instituto Humboldt; Fundación Natura. http://repository. humboldt.org.co/handle/20.500.11761/35369 
Instituto Geográfico Agustín Codazzi (IGAC) (2010). Especificaciones técnicas, cartografia básica. Anexo 3: clasificación de campo para elementos geográficos. IGAC. https://www.igac. gov.co/sites/igac.gov.co/files/anexo3manualdeprocedimientosclasificaciondecampo.pdf

Instituto Geográfico Agustín Codazzi (IGAC) (2013). Estudio semidetallado de suelos: departamento del Quindío. IGAC. https://www.crq.gov.co/2020/images/Info-Ciudadano/ESTUDIO_SEMIDETALLADO_DEL_QUINDIO.pdf

Instituto Geográfico Agustín Codazzi (IGAC) (2014). Mapas de Suelos del Territorio Colombiano a escala 1:100.000. Departamento de Caquetá. Geoportal Datos Abiertos Agrología. https://geoportal.igac.gov.co/contenido/datos-abiertos-agrologia

Instituto Geográfico Agustín Codazzi (IGAC) (2017). Mapas de Clasificación de las Tierras por su Vocación de Uso a escala 1:100.000. Geoportal Datos Abiertos Agrología. https:// geoportal.igac.gov.co/contenido/datos-abiertos-agrologia Instituto Geográfico Agustín Codazzi (IGAC) (2018). Cartografía base: departamento de Caquetá. Geoportal Datos Abiertos Cartografia y Geografia. https://geoportal.igac.gov.co/ contenido/datos-abiertos-cartografia-y-geografia

Kattan, G., Naranjo, L. \& Rojas, V. (2008). Especies focales. En G. Kattan \& L. Naranjo (eds.), Regiones biodiversas: herramientas para la planificación de sistemas regionales de áreas protegidas (pp. 155-166). wCS Colombia; Fundación EcoAndina; wwF Colombia. https://www. researchgate.net/publication/265245902_Regiones_ Biodiversas_Herramientas_para_la_planificacion_ de_sistemas_regionales_de_areas_protegidas

Ley 99 de 1993 (diciembre 22). Por la cual se crea el Ministerio del Medio Ambiente, se reordena el Sector Público encargado de la gestión y conservación del medio ambiente y los recursos naturales renovables, se organiza el Sistema Nacional Ambiental, sinA, y se dictan otras disposiciones. Diario Oficial n 41.146. http://www.secretariasenado.gov.co/senado/basedoc/ley_0099_1993.html

Ministerio de Transporte (2010). Red fluvial nacional de transporte. https://www.invias.gov.co/index.php/archivo-y-documentos/servicios-al-ciudadano/proyectos-invias/610-informe-de-gestion-2010/file

Miranda, R. (2016). Revisión panorámica del uso del pasto Vetiver (Chrysopogon zaizanioides) en restauración de taludes como técnica de bioingeniería del suelo. [Tesis de maestría]. Universidad Jorge Tadeo Lozano. http://hdl.handle. net/20.500.12010/1757
Montoya-Rojas, G. (2018). Lineamientos epistemológicos para la aplicación de la geografia del suelo. Sociedad Geográfica de Colombia.

Montoya-Rojas, G., Almario, M., Bello-Escobar, S., \& Singh, K. (2019). Analysis of the interrelations between biogeographic systems and the dynamics of the Port-Waterfront Cities: Cartagena de Indias, Colombia. Ocean and Coastal Management. doi.org/10.1016/j.ocecoaman. 2019.105055

Montoya-Rojas, G. \& Rivera-Marroquín, M. (2021). Los componentes ambientales de la cuenca Torca: un insumo para el análisis de los escenarios de variabilidad climática en la ciudad de Bogotá, Colombia. Revista U.D.C.A. Actualidad E Divulgación Científica, 24(1). https://doi.org/10.31910/rudca.v24.n1.2021.1833

Moyano-Molano, A. \& Rusinque-Quintero, L. (2020). Conectividad de las áreas protegidas a través del paisaje del departamento de Caquetá. [Tesis de pregrado]. Universidad de Ciencias Aplicadas y Ambientales. https:/ / repository. udca.edu.co/handle/11158/3373

National Research Council (1976). Neotropical Primates: Field Studies and Conservation: Proceedings of a Symposium on the Distribution and Abundance of Neotropical Primates. The National Academies Press. doi.org/10.17226/18666

Organización de las Naciones Unidas para la Alimentación y la Agricultura (FAO) (2008). Base referencial mundial del recurso suelo, un marco conceptual para clasificación, correlación y comunicación internacional. http://www.fao. org/3/a-a0510s.pdf

Palacio, D. (2015). Redes, actores y gobernanza desde un enfoque relacional. En P. Ungar (ed.), Colección Hojas de Ruta. Guías para el estudio socioecológico de la alta montaña en Colombia, libro 2. Instituto de Investigación de Recursos Biológicos Alexander von Humboldt. http://repository.humboldt.org.co/bitstream/ handle/20.500.11761/9294/IAvH_Paramos-Manuales $\% 2002$-web.pdf? sequence $=1$

Payán, E., Moreno, O., Mejía, A., Fonseca, M., \& Carlos, V. (2015). Plan de manejo para la conservación del jaguar (Panthera onca) en el Valle del Cauca, Colombia. Panthera Colombia; Corporación Autónoma Regional del Valle del Cauca. https://www.cvc.gov.co/sites/default/files/Planes_y_Programas/Plan-de-manejo-para-la-conservacion-del-jaguar_Panthera.pdf

Payán, E. \& Soto, C. (2012). Los felinos de Colombia. Ministerio de Ambiente y Desarrollo Sostenible; Instituto de Investigaciones de Recursos Biológicos 
Alexander von Humboldt; Panthera Colombia. http://repository.humboldt.org.co/bitstream/handle $/ 20.500 .11761 / 31413 / 239$.pdf? sequence $=1 \&$ isAllowed $=\mathrm{y}$

Peña-Venegas, G. \& Cardona, G. (2010). Dinámica de los suelos amazónicos: procesos de degradación y alternativas para su recuperación. Instituto Amazónico de Investigaciones Científicas; Ministerio de Ambiente, Vivienda y Desarrollo Territorial. https://www.sinchi.org.co/files/ publicaciones/publicaciones/pdf/librosuelosweb.pdf

Piana, R. (2007). Anidamiento y dieta de Harpia harpyja Linnaeus en la Comunidad Nativa de Infierno, Madre de Dios, Perú. Revista Peruana de Biologia, 14(1), 211-216. http://www.scielo.org.pe/pdf/rpb/v14n1/ v14n01a23.pdf

Rozas, P. \& Figueroa, O. (2006). Conectividad, ámbitos de impacto y desarrollo territorial: análisis de experiencias internacionales. Comisión Económica para América Latina y el Caribe (CEPAL), Naciones Unidas. https://repositorio.cepal.org/bitstream/handle/11362/6314/1/ S0600566_es.pdf

Salazar, C., Riaño, A., Reyes, M., Riaño, U., Castañeda, W., Rubiano, S., \& Rodríguez, C. (2019). Minería: impactos sociales en la Amazonía. Instituto Amazónico de Investigaciones Científicas SINCHI. https://sinchi.org. co/mineria-impactos-sociales-en-la-amazonia

Saura, S. (2013). Conceptos y técnicas para el análisis espacial de datos ecológicos. En M. De la Cruz \& F. Maestre (eds.), Avances en el análisis espacial de datos ecológicos: aspectos metodológicos y aplicados (pp. 1-146). ECESPA-Asociación Española de Ecología Terrestre. http://www. conefor.org/files/usuarios/Capitulo_1_analisis_conectividad_Saura.pdf

Saura, S., González, S., \& Rosselló, R. E. (2011). Evaluación de los cambios en la conectividad de los bosques: el índice de área conexa equivalente y su aplicación en los bosques de Castilla y León. Revista Montes, 3(106), 15-21. http://www.conefor.org/files/usuarios/Saura_et_al_2011_Montes_short_paper_version.pdf

Serje, M. \& Ardila, D. (2017). El río como infraestructura: paisaje y navegación en el río Meta, Colombia. Fronteiras: Fournal of Social, Technological and Environmental Science, 6(1), 95-119. doi.org/10.21664/2238-8869.2017v6il. p95-119

Serrano, D. (2012). Consideraciones en torno al concepto de unidad de paisaje y sistematización de propuestas.
Estudios Geográficos, 73(272), 215-237. doi.org/10.3989/ estgeogr.201208

Shaxson, F. \& Barber, R. (2005). Hidrología, arquitectura del suelo y movimiento del agua. En Optimización de la humedad del suelo para la producción vegetal. El significado de la porosidad del suelo. FAO. https://www.fao.org/3/ y4690s/y4690s06.htm\#bm06

Soil Survey Staff (2014). Keys to Soil Taxonomy. USDA-Natural Resources Conservation Service. https://www. nrcs.usda.gov/wps/portal/nrcs/detail/soils/survey/ class $/$ taxonomy/?cid=nrcs142p2_053580

Stevenson, P. \& Guzmán-Caro, D. (2014). Procesos ecosistémicos provenientes del papel de los monos churucos (Lagothrix lagothricha) como dispersores de semillas. En T. Defler, P. Stevenson, M. Bueno \& D. Guzmán-Caro (eds.), Primates colombianos en peligro de extinción (pp. 332347). doi.org/10.13140/2.1.1808.9605

Taylor, P., Fahrig, L., Henein, K., \& Merriam, G. (1993). Connectivity is a vital element of landscape structure, Oikos, 68(3), 571-573. doi.org/10.2307/3544927

Thót, J. (1963). A Theoretical Analysis of Groundwater Flow in Small Drainage Basins. Geophysical Research, 68(16), 4795-4812. doi.org/10.1029/ Jz068i016p04795

Tocancipá Falla, J. \& Ramírez Castrillón, G. A. (2018). Las nuevas dinámicas rurales en las Zonas de Reserva Campesina en Colombia. Perspectiva Geográfica, 23(1), 31-52. https://doi.org/10.19053/01233769.5796

Unidad de Planificación Rural Agropecuaria (UPRA) (2014). Presentación institucional. https://www.minagricultura. gov.co/Documents/UPRA_Oferta_Institucional.pdf

Vásquez, J. \& Andrade, G. (2016). El paisaje protegido, pieza faltante en el Sistema de Áreas Protegidas de Colombia. Biodiversidad en la Práctica, 1(1), 128-146. http://revistas.humboldt.org.co/index.php/BEP/article/view/49/49

Vila, J., Varga, D., Llausàs, A., \& Ribas, A. (2006). Conceptos y métodos fundamentales en ecología del paisaje (landscape ecology). Una interpretación desde la geografía. Documents d'Anàlisi Geogràfica, 48, 151-166. https:// www.raco.cat/index.php/DocumentsAnalisi/article/ view/72657

Von Bertalanffy, L. (1993). General System Theory: Foundations, Development, Applications. George Braziller. https:// monoskop.org/images/7/77/Von_Bertalanffy_Ludwig_General_System_Theory_1968.pdf 
Wu, J. (2019). Landscape ecology. In Encyclopedia of Ecology (vol. 4, pp. 527-531). doi.org/10.1016/B978-0-12409548-9.10919-4
Zubelzu, S. \& Allende, F. (2015). El concepto de paisaje y sus elementos constituyentes: requisitos para la adecuada gestión del recurso y adaptación de los instrumentos legales en España. Revista Colombiana de Geografia, 24(1), 29-42. doi.org/10.15446/rcdg.v24n1.41369 